\title{
Imaging chemical differentiation around the low-mass protostar L483-mm
}

\author{
J. K. Jørgensen
}

\author{
Leiden Observatory, PO Box 9513, 2300 RA Leiden, The Netherlands \\ e-mail: joergensen@strw.leidenuniv.nl
}

Received 11 February 2004 / Accepted 14 May 2004

\begin{abstract}
This paper presents a millimeter wavelength aperture-synthesis study of the spatial variations of the chemistry in the envelope around the deeply embedded low-mass protostar L483-mm on $1000 \mathrm{AU}\left(5^{\prime \prime}\right)$ scales. Lines of 8 molecular species including $\mathrm{CN}, \mathrm{C}^{18} \mathrm{O}, \mathrm{CS}, \mathrm{C}^{34} \mathrm{~S}, \mathrm{HCN}, \mathrm{H}^{13} \mathrm{CN}, \mathrm{HCO}^{+}$and $\mathrm{N}_{2} \mathrm{H}^{+}$have been observed using the Owens Valley Radio Observatory Millimeter Array. Continuum emission at $2.7-3.4 \mathrm{~mm}$ is well-fit by an envelope model based on previously reported submillimeter continuum images down to the sensitivity of the interferometer without introducing a disk/compact source, in contrast to what is seen for other protostellar objects. A velocity gradient in dense material close to the central protostar is traced by $\mathrm{HCN}, \mathrm{CS}$ and $\mathrm{N}_{2} \mathrm{H}^{+}$, and is perpendicular to the large-scale $\mathrm{CO}$ outflow, with a pattern consistent with rotation around a $\sim 1 M_{\odot}$ central object. Velocity gradients in the propagation direction of the outflow suggest a clear interaction between the outflowing material and "quiescent" core. Significant differences are observed between the emission morphologies of various molecular species. The $\mathrm{C}^{18} \mathrm{O}$ interferometer observations are fit with a "drop" abundance profile where $\mathrm{CO}$ is frozen-out in a region of the envelope with temperatures lower than $40 \mathrm{~K}$ and densities higher than $1.5 \times 10^{5} \mathrm{~cm}^{-3}$, which is also required to reproduce previously reported single-dish observations. The $\mathrm{N}_{2} \mathrm{H}^{+}$emission strongly resembles that of $\mathrm{NH}_{3}$ and is found to be absent toward the central continuum source. This is a direct consequence of the high $\mathrm{CO}$ abundances in the inner region as illustrated by a chemical model for the $\mathrm{L} 483$ envelope. The observed $\mathrm{CN}$ emission forms a spatial borderline between the outflowing and quiescent material probed by, respectively, $\mathrm{HCO}^{+}$and $\mathrm{N}_{2} \mathrm{H}^{+}$, and also shows intermediate velocities compared to these two species. A scenario is suggested in which $\mathrm{CN}$ is enhanced in the walls of an outflow cavity due to the impact of UV irradiation either from the central protostellar system or related to shocks caused by the outflow.
\end{abstract}

Key words. ISM: individual objects: L483-mm - stars: formation - ISM: molecules - ISM: abundances - astrochemistry

\section{Introduction}

The chemistry of star-forming regions shows a richness and complexity reflecting large variations in the physical conditions found in these environments. For example, the thermal evolution of the pre- and protostellar cores results in evaporation and freeze-out of molecules illustrating the important interplay between the solid-state and gas-phase chemistry. Recent singledish surveys of low-mass protostars (Jørgensen et al. 2002, 2004c; Schöier et al. 2002; Maret et al. 2004) have illustrated that the chemistry may be severely affected by the thermal history of cores, with radial variations of the temperature due to the heating from the central protostar. This paper presents highresolution millimeter wavelength aperture-synthesis observations of a wide range of different molecules toward the embedded low-mass protostar L483-mm. In combination with a detailed radiative transfer model (Jørgensen et al. 2002, 2004c) these high-resolution observations make it possible to resolve and address the spatial variation of the chemistry in the protostellar envelope on $\sim 1000$ AU (5") scales. Only a few protostars have previously been studied in this chemical detail and the combination with line radiative transfer models and information about the larger scale structure from single-dish observations makes a unique discussion about the chemical differentiation at varying temperatures and densities possible.

Recent single-dish continuum studies of embedded protostars have proved useful to establish the physical structure of the protostellar envelopes. A detailed description of the density and temperature variation is crucial for calculations of the molecular excitation and for the interpretation of the chemical small-scale structure from high resolution interferometer observations. Typically the envelope density and temperature structure can be constrained from radiative transfer modeling of submillimeter continuum observations (e.g., Jørgensen et al. 2002; Shirley et al. 2002) or from infrared extinction measurements (e.g., Alves et al. 2001; Harvey et al. 2001). The detailed profiles can subsequently be used as input for Monte Carlo radiative transfer modeling of single-dish line emission to constrain the molecular abundances (e.g., Bergin et al. 2002; Jørgensen et al. 2002, 2004c; Schöier et al. 2002). The models can also be used to predict images of both continuum and 
Table 1. Parameters for L483 from Jørgensen et al. (2002).

\begin{tabular}{ll}
\hline \hline Distance, $d$ & $200 \mathrm{pc}$ \\
$L_{\mathrm{bol}}$ & $9 L_{\odot}$ \\
$T_{\text {bol }}$ & $50 \mathrm{~K}$ \\
\hline Envelope parameters: & \\
\hline Inner radius $(T=250 \mathrm{~K}), R_{i}$ & $9.9 \mathrm{AU}$ \\
Outer radius, $R_{10 \mathrm{~K}}$ & $1.0 \times 10^{4} \mathrm{AU}$ \\
Density at $1000 \mathrm{AU}, n\left(\mathrm{H}_{2}\right)$ & $1.0 \times 10^{6} \mathrm{~cm}^{-3}$ \\
Slope of density distribution, $p$ & 0.9 \\
Mass, $M_{10 \mathrm{~K}}$ & $4.4 M_{\odot}$ \\
\hline
\end{tabular}

line emission that can be directly compared to interferometer observations constraining the envelope structure down to scales of a few hundred AU (e.g., Jørgensen et al. 2004a; Schöier et al. 2004). The caveat of the single-dish studies is their low spatial resolution of $10-15^{\prime \prime}$ or worse, which is why these observations predominantly are sensitive to material on larger scales ( $\gtrsim 2000 \mathrm{AU}$ ) unless high-excitation lines are observed. Recent interferometric studies of millimeter continuum emission around low-mass protostars (e.g., Hogerheijde et al. 1999; Looney et al. 2000; Harvey et al. 2003; Jørgensen et al. 2004a; Schöier et al. 2004) have illustrated the potential for probing the small-scale physical structure of the envelopes and constrain the presence of unresolved emission, possibly originating in circumstellar disks in these deeply embedded stages.

Images of molecular line emission at similar resolutions can be used to discuss the detailed envelope chemical structure. For example, Jørgensen et al. (2004a) reported highresolution observations of a range of molecular species toward the class 0 protostar, NGC 1333-IRAS 2A. The 3-6" (600$1200 \mathrm{AU})$ observations were interpreted in the context of envelope models constrained by single-dish continuum maps and multi-transition line observations from the survey by Jørgensen et al. $(2002,2004 c)$. It was found that the single-dish envelope model could be successfully extrapolated to the smaller scales, lending further credibility to the approach and derived physical and chemical properties.

L483-mm (IRAS 18148-0440; in the following just L483) is similar to NGC 1333-IRAS 2A being a deeply embedded, low-mass protostar with a low bolometric temperature of $\approx 50 \mathrm{~K}$. In contrast to NGC 1333-IRAS $2 \mathrm{~A}$, it shows a remarkable, asymmetric structure in the SCUBA maps. This asymmetry is likely to be the cause of the rather flat density distributions $\left(n \propto r^{-p}\right)$ found in radiative transfer modeling of the continuum emission by Jørgensen et al. (2002) ( $p=0.9)$ and Shirley et al. (2002) $(p=1.2)$. The envelope parameters for $L 483$ are given in Table 1 .

Park et al. (2000) reported BIMA observations of $\mathrm{HCO}^{+} 1-0$ and $\mathrm{C}_{3} \mathrm{H}_{2} \quad 2_{12}-1_{01}$ toward L483. They found two characteristic velocity gradients in the two species: an eastwest velocity gradient in $\mathrm{HCO}^{+}$coincident with the larger scale $\mathrm{CO}$ outflow and a north-south velocity gradient in $\mathrm{C}_{3} \mathrm{H}_{2}$ which they associated with global contraction of an envelope of a few thousand AU size. L483 has also been mapped in $\mathrm{NH}_{3}$, most recently by Fuller \& Wootten (2000) who presented $\mathrm{NH}_{3}$ maps from the VLA. They found that the overall asymmetry observed with SCUBA also shows up in the $\mathrm{NH}_{3}$ maps, but with $\mathrm{NH}_{3}$ lacking emission close to the protostar, which they ascribed to optical depth effects. They also noted a characteristic velocity pattern across the maps and suggested the presence of infall close to the central protostar.

In addition to clear signatures in $\mathrm{CO}$ emission (Fuller et al. 1995; Hatchell et al. 1999; Tafalla et al. 2000), an outflow driven by L483 is also seen through near-infrared emission, possibly caused by scattered emission in the outflow cavities (Hodapp 1994; Fuller et al. 1995). The absence of significant enhancements of $\mathrm{CH}_{3} \mathrm{OH}$ and $\mathrm{SiO}$, as found in other outflows, led Tafalla et al. (2000) to suggest that the L483 outflow is more evolved than those from other class 0 objects. Since the driving source itself appears deeply embedded (Fuller et al. 1995; Fuller \& Wootten 2000), Tafalla et al. therefore suggested that L483 is in transition from the class 0 to the class I stage.

This paper presents high resolution $3 \mathrm{~mm}$ interferometer observations of a wide range of molecular species toward L483. The main objective is to address whether the observed trends in the single-dish survey by Jørgensen et al. (2004c) can be related to the variations in the chemistry in the L483 envelope. The paper is laid out as follows: Sect. 2 describes the details of the observations. Section 3 discusses the continuum maps of L483 and compares to the predictions from the envelope model based on the SCUBA observations from Jørgensen et al. (2002). Section 4 presents the line observations. Section 5 brings together the results for the line emission and discusses the implications for the chemistry in the environment of L483.

\section{Observations}

L483-mm $\left(\alpha_{2000}=18^{\mathrm{h}} 17^{\mathrm{m}} 29^{\mathrm{s}} .8, \delta_{2000}=-04^{\circ} 39^{\prime} 38^{\prime \prime} .3\right)$ was observed using the Owens Valley Radio Observatory (OVRO) Millimeter Array ${ }^{1}$ from October 2001 to February 2003 in three settings including $\mathrm{HCN}, \mathrm{H}^{13} \mathrm{CN}$ and $\mathrm{HCO}^{+}(3.4 \mathrm{~mm})$, $\mathrm{N}_{2} \mathrm{H}^{+}, \mathrm{CS}$ and $\mathrm{C}^{34} \mathrm{~S}(3.2 \mathrm{~mm})$, and $\mathrm{C}^{18} \mathrm{O}$ and $\mathrm{CN}(2.7 \mathrm{~mm})$ as summarized in Table 2. Each of the settings was observed in the $\mathrm{C}$ and $\mathrm{E}$ configurations providing projected baselines ranging from 3 to $45 \mathrm{k} \lambda$. Compared to the $\mathrm{BIMA} \mathrm{HCO}^{+}$maps presented by Park et al. (2000), the $(u, v)$ coverage of the tracks in this paper includes longer baselines but not the shortest spacings. These observations are therefore less sensitive to extended emission but provide higher resolution. The correlator gives spectral resolutions of $\approx 0.15-0.2 \mathrm{~km} \mathrm{~s}^{-1}$ over 128 channels covering each line with rms noise levels of $0.1 \mathrm{Jy}^{\text {beam }}{ }^{-1}$ per channel. The complex gains were calibrated by regular observations of the nearby quasar, j1743-038, approximately every 20-25 min. The bandpass was calibrated by observations of strong quasars, the fluxes using the same quasars as secondary calibrators and Uranus and Neptune as primary calibrators. Calibration of the data was performed using the MMA reduction package (Scoville et al. 1993).

\footnotetext{
1 The Owens Valley Millimeter Array is operated by the California Institute of Technology under funding from the US National Science Foundation (grant No. AST-9981546).
} 
Table 2. Summary of the observed lines.

\begin{tabular}{llc}
\hline \hline Molecule & Transition & Frequency [GHz] \\
\hline $\mathrm{H}^{13} \mathrm{CN}$ & $1-0^{a}$ & 86.3402 \\
$\mathrm{HCN}$ & $1-0^{a}$ & 88.6318 \\
$\mathrm{HCO}^{+}$ & $1-0$ & 89.1885 \\
$\mathrm{~N}_{2} \mathrm{H}^{+}$ & $1-0^{a}$ & 93.1737 \\
$\mathrm{C}^{34} \mathrm{~S}$ & $2-1$ & 96.4129 \\
$\mathrm{CS}$ & $2-1$ & 97.9810 \\
$\mathrm{C}^{18} \mathrm{O}$ & $1-0$ & 109.7822 \\
$\mathrm{CN}$ & $1-0^{a}$ & 113.4910 \\
\hline
\end{tabular}

Notes: ${ }^{a}$ Hyperfine splitting observed.

\section{Continuum emission}

The continuum maps clearly show the central protostar close to the phase center (Fig. 1). The emission appears resolved and roughly follows the morphology from the larger scale SCUBA maps. Results of Gaussian fits to the visibility data are given in Table 3. The envelope model of Jørgensen et al. (2002) predicts total integrated fluxes ranging from 0.16 to $0.32 \mathrm{Jy}$ at wavelengths from 3.4 to $2.6 \mathrm{~mm}$. Figure 2 shows that the envelope model reproduces the observed fluxes on all the baselines, but the total flux recovered in the interferometer maps is only $5-10 \%$ of the total model flux. This is likely due to the lack of sensitivity in the interferometer observations to extended emission.

From Fig. 2 it is also evident that very little emission is picked up on baselines longer than 20-25 $\mathrm{k} \lambda$ (i.e., at scales less than $\left.10^{\prime \prime}\right)$. Figure 3 shows the fitted point source fluxes after subtracting the envelope model ( $F_{\text {compact }}$, listed in Table 3 ). It can be seen that the emission is consistent with the zeroexpectation level (the expected amplitude signal due to noise alone in the absence of source emission) within $1 \sigma$ (3.2 and $3.4 \mathrm{~mm}$ observations $)$ and $2 \sigma(2.6 \mathrm{~mm})$. This contrasts the situation for NGC 1333-IRAS 2A (Jørgensen et al. 2004a) and IRAS 16293-2422 and L1448-C (Schöier et al. 2004) which show compact emission not explained by the envelope models. In fact the upper limit to the compact flux at $2.6 \mathrm{~mm}$ restricts the mass of a possible disk to $\lesssim 0.04 M_{\odot}$ assuming a temperature of $30 \mathrm{~K}$ and optically thin emission. Radio measurements of L483 at centimeter wavelengths (Beltrán et al. 2001) show a central source of $0.20 \mathrm{mJy}(6 \mathrm{~cm})$ and $0.31 \mathrm{mJy}(3.6 \mathrm{~cm})$. These radio observations are compared to the millimeter data from this paper in the insert in Fig. 3. As can be seen, a spectral index consistent with optically thin emission cannot simultaneous explain the millimeter and centimeter observations; however, a flat, positive spectral index would be consistent with the emission at centimeter wavelengths and the limits on the millimeter observations. This would be the case if the flux from centimeter to millimeter wavelengths is from thermal free-free emission as suggested by Beltrán et al. (2001).
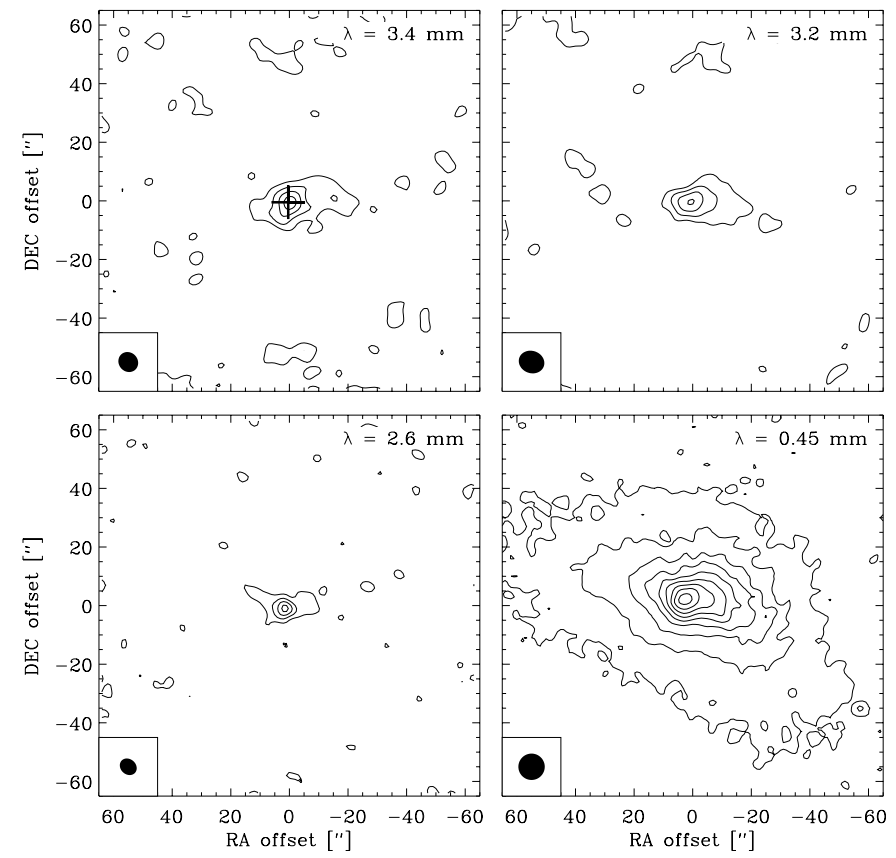

Fig. 1. Continuum maps at $\lambda=3.4,3.2$ and $2.6 \mathrm{~mm}$ from the OVRO observations compared to the SCUBA $450 \mu \mathrm{m}$ map. The contours are in levels of $2 \sigma$ as given in Table 3 . The position of the radio source from Beltrán et al. (2001) is indicated by the black "+" in the upper left, $\lambda=3.4 \mathrm{~mm}$, map.

\section{Line emission}

\subsection{Morphology}

Maps of the total integrated emission of each of the observed lines are shown in Fig. 4. All species are detected toward the continuum position and most are stretched in the east-west direction of the elongated core from the SCUBA maps.

The $\mathrm{N}_{2} \mathrm{H}^{+}$emission is seen to follow the contours of the dust emission from the SCUBA continuum maps closely, except toward the position of the central star where the emission appears suppressed. This gives $\mathrm{N}_{2} \mathrm{H}^{+}$emission a unique "peanut-shape" compared to the remaining species, with the strongest emission at two lobes, respectively east and southwest of the continuum peak position. $\mathrm{C}^{18} \mathrm{O}$ in contrast is mainly seen very closely confined to the region around the central protostar. The emission is resolved and interestingly shows an almost triangular shape similar to that found in the SCUBA maps (e.g., lower right panel of Fig. 1): the two shortest sides in the north-south direction on the western side of the core and in the east-west direction on the northern side of the core, and the longest side stretching from the northeast to the southwest.

$\mathrm{HCO}^{+}$shows the most extended emission. It has a slightly curved structure around the central protostar, being almost anticorrelated with the $\mathrm{N}_{2} \mathrm{H}^{+}$emission. A similar "twist" can be seen in the single-dish CO maps of Hatchell et al. (1999) and Tafalla et al. (2000) and in general the $\mathrm{HCO}^{+}$appears closely correlated with the outflow, as was also concluded based on the BIMA $\mathrm{HCO}^{+}$maps by Park et al. (2000). Figure 5 compares the 


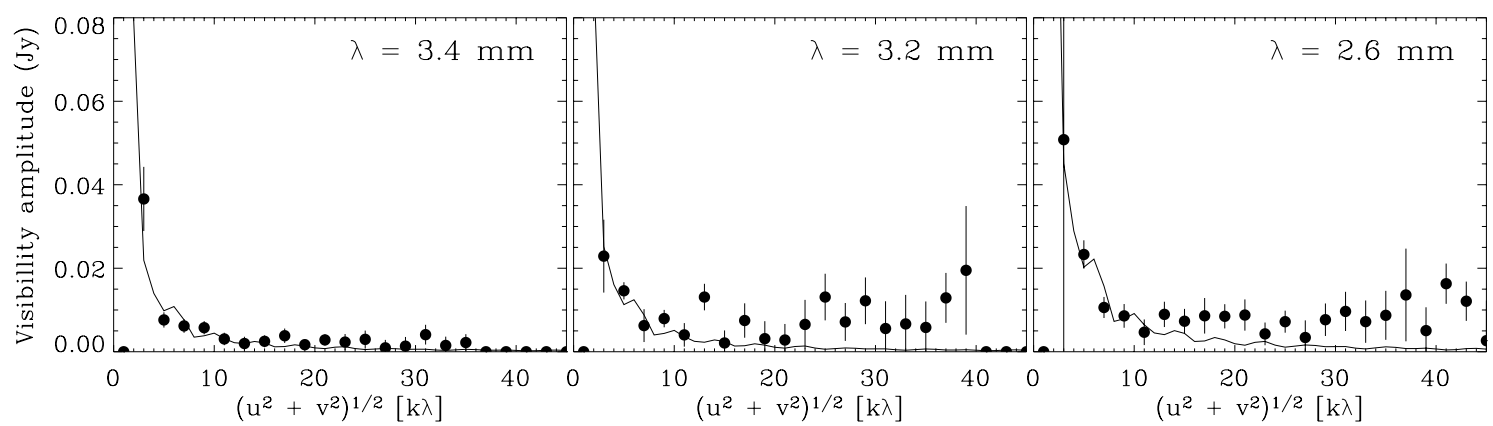

Fig. 2. Plots of visibility amplitudes vs. projected baseline line length for the three continuum datasets at $\lambda=3.4,3.2$ and $2.6 \mathrm{~mm}$. The solid circles indicate the observations and the lines the predictions from the model for the L483 envelope.

Table 3. Results from fitting Gaussians to the visibility data for the three continuum datasets.

\begin{tabular}{llll}
\hline \hline Wavelength [mm] & 3.4 & 3.2 & 2.6 \\
\hline rms [mJy beam $^{-1}$ ] & 0.5 & 0.9 & 0.8 \\
Beam size $(\mathrm{HPBW})\left[{ }^{\prime \prime}\right]$ & $7.1 \times 6.5$ & $8.8 \times 7.6$ & $6.2 \times 5.2$ \\
$F_{\text {total }}[\mathrm{mJy}]$ & $13 \pm 2$ & $12 \pm 2$ & $11 \pm 2$ \\
$X$-offset ["] & $-0.5 \pm 0.9$ & $0.1 \pm 0.8$ & $1.5 \pm 0.3$ \\
$Y$-offset ["] & $-0.9 \pm 0.7$ & $-0.3 \pm 0.5$ & $-0.9 \pm 0.3$ \\
$F_{\text {compact }}{ }^{a}[\mathrm{mJy}]$ & $1.0 \pm 0.5(2.0)$ & $4.8 \pm 1.0(5.2)$ & $5.0 \pm 0.9(4.5)$ \\
\hline
\end{tabular}

${ }^{a}$ Fitted emission from a compact source after subtraction of the model prediction for the envelope emission. The numbers in parentheses indicate the zero-expectation level, i.e., the expected amplitude signal due to noise alone in the absence of any source.

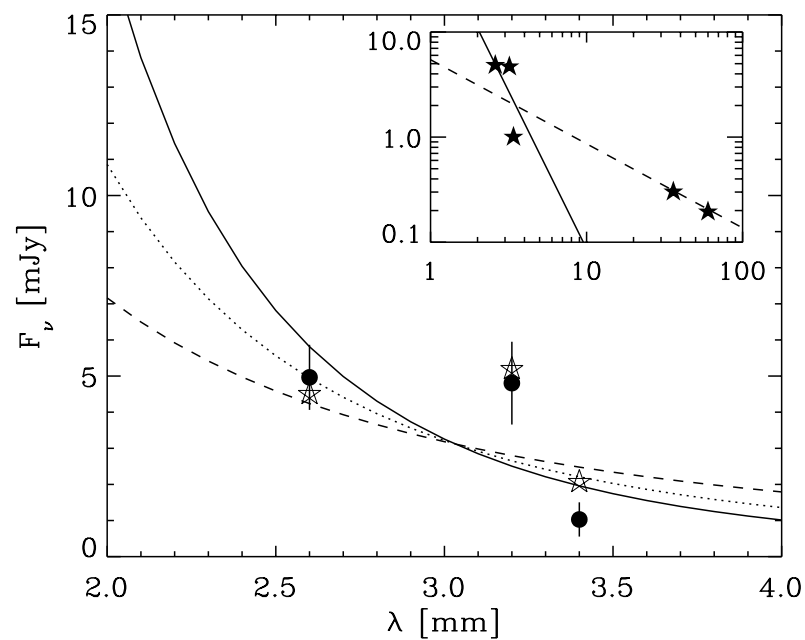

Fig. 3. Compact (unresolved) emission as a function of wavelength at 2.6, 3.2 and $3.4 \mathrm{~mm}$. For each data point the expected flux in the absence of any point source is indicated by an open star. Fitted powerlaw distributions with spectral indexes of 2, 3 and 4 are shown with the dashed, dotted and solid lines, respectively. The insert show the millimeter fluxes compared to the centimeter data from Beltrán et al. (2001). Here the dashed line indicates the flux from centimeter wavelength with a spectral index of 0.8 . The solid line indicates a spectral index of 3, corresponding to optically thin emission from dust with opacities $\kappa_{v} \propto \nu^{\beta}$ where $\beta=1$ at millimeter wavelengths.

2MASS All-Sky Quicklook ${ }^{2} K_{\mathrm{s}}$ image of L483 with the observed $\mathrm{HCO}^{+}$emission. Near-perfect overlap is seen between the

\footnotetext{
2 The Two Micron All Sky Survey (2MASS) is a joint project of the University of Massachusetts and the Infrared Processing and
}

infrared and $\mathrm{HCO}^{+}$emission in the western outflow lobe. This in agreement with the suggestion by Fuller et al. (1995) that the infrared emission is light from the central protostar scattered off the outflow cavity walls. The HCN and CS emission appear to be more centrally concentrated around the central protostar but do show traces of the same elongated east-west feature. In addition a north-south feature perpendicular to the main elongation is seen in both species extending over $\approx 20^{\prime \prime}$. Their weaker isotopic species, $\mathrm{C}^{34} \mathrm{~S}$ and $\mathrm{H}^{13} \mathrm{CN}$, only show unresolved emission around the central continuum peak.

$\mathrm{CN}$ shows a very narrow feature elongated in the east-west direction. Toward both $\mathrm{N}_{2} \mathrm{H}^{+}$lobes the $\mathrm{CN}$ emission makes a twist as seen in the $\mathrm{HCO}^{+}$maps, but for $\mathrm{CN}$ the emission is much narrower. In fact $\mathrm{CN}$ is found to trace the boundary between the $\mathrm{N}_{2} \mathrm{H}^{+}$and $\mathrm{HCO}^{+}$emission (see Sect. 5.2). The two $\mathrm{CN}$ hyperfine components otherwise show similar structures indicating quite homogeneous excitation conditions along the main elongation.

As found for the continuum observations, the interferometer picks up only a fraction of the line emission seen by the single-dish telescope. Figure 6 compares the single-dish spectra from the Onsala $20 \mathrm{~m}$ telescope (Jørgensen et al. 2002, $2004 \mathrm{c}$ ) with those from the interferometer datacubes convolved with Gaussians similar to the size of the single-dish beam.

Analysis Center/California Institute of Technology, funded by the National Aeronautics and Space Administration and the National Science Foundation. The presented image was obtained from the NASA/IPAC Infrared Science Archive, which is operated by the Jet Propulsion Laboratory, California Institute of Technology, under contract with the National Aeronautics and Space Administration. 


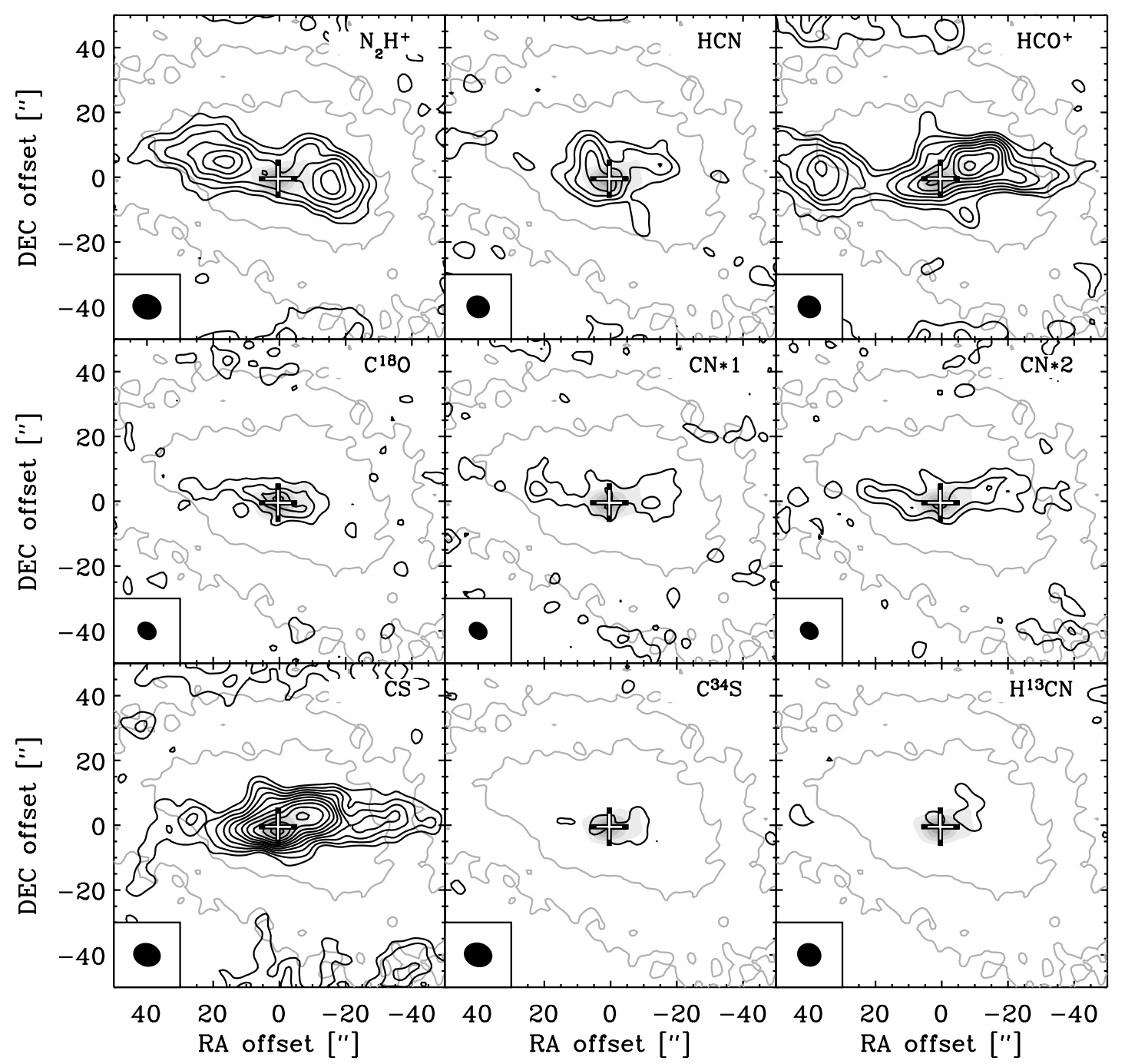

Fig. 4. Overview of all observed lines at OVRO. The black line contours indicate the line emission integrated from 2.5 to $8.0 \mathrm{~km} \mathrm{~s}^{-1}$ for CS, $\mathrm{HCN}, \mathrm{HCO}^{+}$, and $\mathrm{N}_{2} \mathrm{H}^{+}$and from 4.0 to $6.5 \mathrm{~km} \mathrm{~s}^{-1}$ for the remaining species with levels given in steps of $2 \sigma$. The grey line contours indicate the SCUBA $450 \mu \mathrm{m}$ emission, the grey-scale map indicates the $3.4 \mathrm{~mm}$ continuum emission. For $\mathrm{HCN}, \mathrm{H}^{13} \mathrm{CN}$ and $\mathrm{N}_{2} \mathrm{H}^{+}$the emission is integrated over the main hyperfine component. The $\mathrm{CN} * 1$ and $\mathrm{CN} * 2$ maps show the emission of the two $\mathrm{CN} 1-0$ hyperfine components at 113.489 and $113.491 \mathrm{GHz}$, respectively.

The interferometer spectra recover only a fraction of the emission close to the systemic velocity of the cloud $\left(\approx 5.3 \mathrm{~km} \mathrm{~s}^{-1}\right)$ whereas that in the wings agrees better, i.e., is less subject to resolving out.

The large degree of resolving out, together with the asymmetry of the source, naturally complicates the interpretation of the line observations and, in particular, renders direct calculations of, e.g., column densities based on the absolute values of the interferometer observations impossible. The interferometer observations do, however, give the location and sizes of the brightest emission and one can thereby utilize comparisons between the maps of the different species to address the spatial variations of the chemistry. Also, predictions from the line radiative transfer model can be used for comparison to the interferometer observations, especially for species that show a relatively simple structure such as $\mathrm{C}^{18} \mathrm{O}$ (see discussion in Sect. 5.1).

\subsection{Velocity field}

Velocity gradients are seen for a number of species toward $\mathrm{L} 483$ as illustrated in channel maps of the lines of $\mathrm{HCO}^{+}, \mathrm{HCN}$, $\mathrm{CS}$ and $\mathrm{N}_{2} \mathrm{H}^{+}$in Fig. 7. The $\mathrm{HCO}^{+}$maps clearly show the twist of the outflow toward the east for the red-shifted lobe, with distinct cores at offsets $\left(15^{\prime \prime},-5^{\prime \prime}\right)$ at $6.6 \mathrm{~km} \mathrm{~s}^{-1}$ and the edge of the channel maps at $>6.6 \mathrm{~km} \mathrm{~s}^{-1}$. The first of these two clumps are also clearly seen in the CS channel maps at the same velocity. This core is merging with the central protostellar core at offsets $\left(3^{\prime \prime},-3^{\prime \prime}\right)$ seen toward all four species. In the maps of the blue-shifted emission the northern part of the central core is also seen at $\left(0^{\prime \prime}, 5^{\prime \prime}\right)$ for velocities around $4.2 \mathrm{~km} \mathrm{~s}^{-1}$. The outflow emission is less obvious in the blue-shifted lobe. A last feature is a red-shifted core at $\left(-15^{\prime \prime}, 0^{\prime \prime}\right)$ in $\mathrm{HCN}, \mathrm{HCO}^{+}$and $\mathrm{CS}$ at velocities of $6.3 \mathrm{~km} \mathrm{~s}^{-1}$. The two peaks of the $\mathrm{N}_{2} \mathrm{H}^{+}$emission are seen to be unrelated to emission in the other maps and peak at respectively 5.1 and $6.3 \mathrm{~km} \mathrm{~s}^{-1}$. 


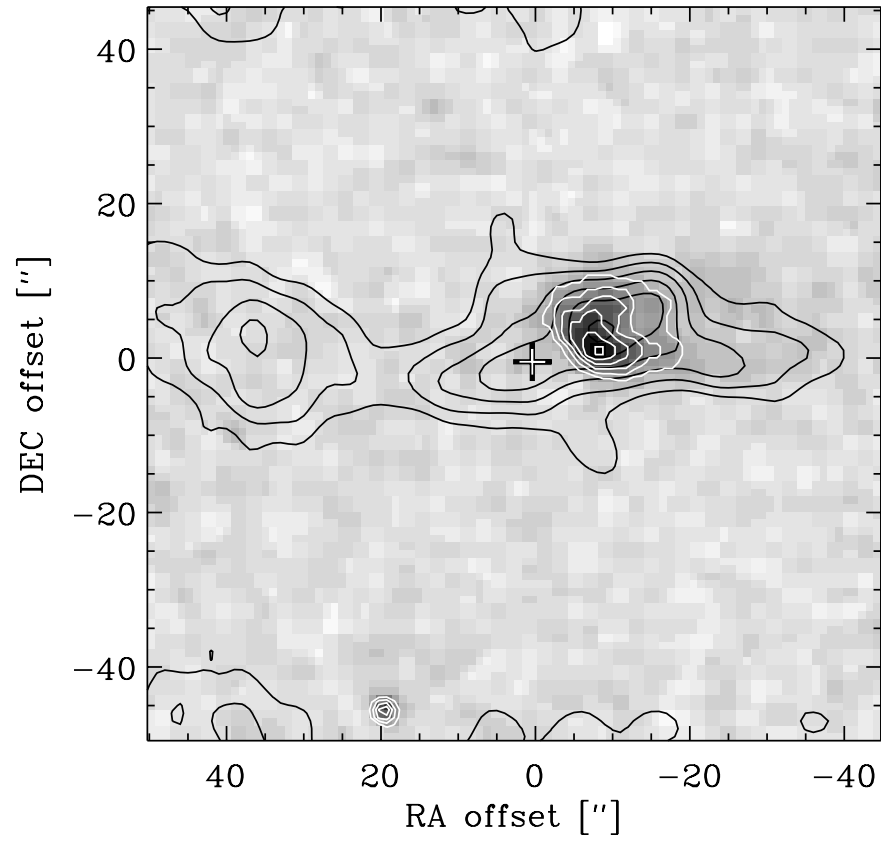

Fig. 5. 2MASS near-infrared $K_{\mathrm{s}}$ image of L483 (grey-scale and white contours) compared to the integrated $\mathrm{HCO}^{+} 1-0$ emission (black contours; in steps of $3 \sigma$ ). Notice the near-perfect agreement between the infrared nebulosity and the outflow emission probed by $\mathrm{HCO}^{+}$.

As discussed by Jørgensen et al. (2004a), it may be somewhat problematic directly inferring velocity gradients from interferometer maps with missing short-spacings since the interferometer is predominantly sensitive to material at the more extreme velocities. As can be seen from Fig. 6, the emission in the line wings is indeed least subject to resolving out compared to the emission close to the systemic velocity, but in general the observed line profiles appear similar from both single-dish and interferometer observations indicating that they are still probing much the same material.

For $\mathrm{N}_{2} \mathrm{H}^{+}$, the hyperfine splitting further complicates the interpretation of the velocity field. As was also found for NGC 1333-IRAS 2A, however, each component is rather narrow, not affected by the outflow, and well-represented by single Gaussians. To derive the velocity field probed by $\mathrm{N}_{2} \mathrm{H}^{+}$, the emission from the 7 hyperfine components was therefore fit simultaneously to the spectra in each pixel. The linewidths for the individual hyperfine components were taken to be constant and fit together with the systemic velocity field, the overall normalization of the entire hyperfine group and the relative intensities of the hyperfine components. The fitted velocity field is illustrated in Fig. 8. An overall red-blue asymmetry is found between the eastern and western lobes. Toward the source position it is seen that the velocity gradient is directed toward the north-south as in the cases of HCN and CS.

The very characteristic two lobes in $\mathrm{N}_{2} \mathrm{H}^{+}$are also seen in $\mathrm{NH}_{3}$ by Fuller \& Wootten (2000), who furthermore found that the $\mathrm{NH}_{3}$ emission shows a two velocity component structure with the NE lobe red-shifted to $5.5-5.6 \mathrm{~km} \mathrm{~s}^{-1}$ and the SW lobe blue-shifted to $5.3-5.4 \mathrm{~km} \mathrm{~s}^{-1}$. This is exactly what is found for our $\mathrm{N}_{2} \mathrm{H}^{+}$data, indicating that the $\mathrm{NH}_{3}$ and $\mathrm{N}_{2} \mathrm{H}^{+}$emission probe the same material. Both Fuller et al. (1995) and
Tafalla et al. (2000) proposed L483 as a core being broken up by the action of the outflow resulting, for example, in the bipolar near-infrared nebulosity. The velocity field seen in $\mathrm{N}_{2} \mathrm{H}^{+}$ and $\mathrm{NH}_{3}$ and its similarities with the outflow velocity pattern would suggest that such an interaction in fact is ongoing although these species are not directly probing the outflowing gas.

Fuller \& Wootten (2000) found the most blue-shifted emission toward the central star with velocities of $5.1-5.3 \mathrm{~km} \mathrm{~s}^{-1}$, and suggested that the more blue-shifted emission toward the central star is a result of infall. In our $\mathrm{N}_{2} \mathrm{H}^{+}$maps it is also seen that the emission has its most blue-shifted peak about $5^{\prime \prime}$ northwest of the protostellar $\mathrm{mm} / \mathrm{cm}$ source. However, an accompanying red-shifted component is present on the other side of the central source (i.e., to the southeast). This is further supported by our HCN and CS maps which show a similar velocity pattern around the central protostar, also seen by Park et al. (2000) in $\mathrm{C}_{3} \mathrm{H}_{2}$ maps. A tempting suggestion for this velocity gradient is rotation around the central protostellar object and the propagation axis of the outflow. The $\mathrm{CS}$ and $\mathrm{HCN}$ lines have rather high critical densities and appear to probe only the densest gas on small scales close to the central protostar. Likewise $\mathrm{C}_{3} \mathrm{H}_{2}$ is typically only found in the dense envelope material not affected by the outflow (e.g., Bachiller \& Pérez Gutiérrez 1997; Tafalla \& Myers 1997).

Figure 9 shows the position-velocity diagram for the $\mathrm{CS}$ emission. The coordinate system has been rotated to have the $Y$-axis in the direction of the north-south velocity gradient around the central protostar (i.e., $12^{\circ}$ from north through east) and the $X$-axis in the direction of the outflow perpendicular to this. The largest velocity gradient is seen in the north-south direction of the $\mathrm{CO}$ outflow. At velocities $\sim 1 \mathrm{~km} \mathrm{~s}^{-1}$ from the systemic velocity the gradient is found to be consistent with linear expansion of $0.27 \mathrm{~km} \mathrm{~s}^{-1} \operatorname{arcsec}^{-1}$ or $2.6 \times 10^{-4} \mathrm{~km} \mathrm{~s}^{-1} \mathrm{pc}^{-1}$ at the distance (200 pc) of L483.

A clear velocity gradient is also observed in the north-south direction: plotted on top of the position-velocity diagram is the predicted rotation curve for Keplerian rotation around a 1.2 $M_{\odot}$ central object. This velocity gradient fits the data at large velocities compared to the systemic velocity, but at velocities of $4.5-5 \mathrm{~km} \mathrm{~s}^{-1}$ the emission appears to stretch southwards in contrast to the prediction for Keplerian rotation. The cause of this is the offset in the north-south direction between the red and blue-shifted lobes of the outflow. Alternative explanations to the pure Keplerian rotation (e.g., a combination of rotation and infall such as suggested for another embedded low-mass YSO, TMC1, by Hogerheijde 2001) cannot be ruled out based on the data presented in this paper, since the confusion with the outflow around L483 complicates the interpretation.

\section{Discussion}

\subsection{Thermal structure, depletion of $\mathrm{CO}$ and resulting chemistry}

The most striking feature in the maps of the integrated emission in Fig. 4 is the characteristic "peanut-shaped" $\mathrm{N}_{2} \mathrm{H}^{+}$maps, with $\mathrm{N}_{2} \mathrm{H}^{+}$being absent from the gas-phase close to the 


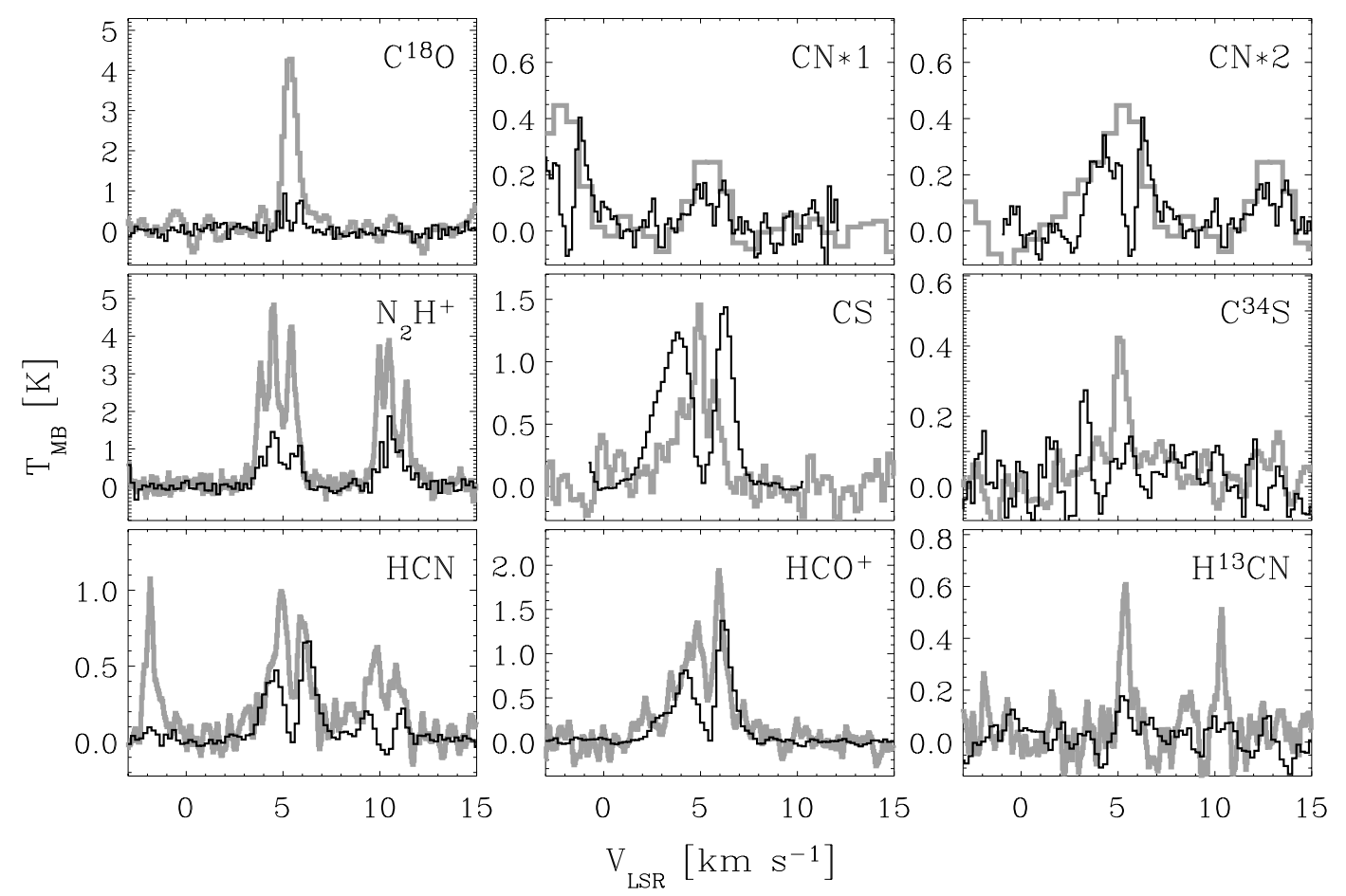

Fig. 6. Comparison between the single-dish spectra (grey) and spectra extracted from the interferometer datacubes convolved with the singledish beam (black). Each spectrum from the interferometer data has furthermore been multiplied by a factor 5 to make comparison between the lineshapes easier. As in Fig. 4 the spectra marked $\mathrm{CN} * 1$ and $\mathrm{CN} * 2$ shows the emission of the two CN 1-0 hyperfine components at 113.489 and $113.491 \mathrm{GHz}$, respectively.

central protostar. This likely reflects destruction of $\mathrm{N}_{2} \mathrm{H}^{+}$by reactions with $\mathrm{CO}$, as suggested to be the case in protostellar environments by, e.g., Bergin et al. (2001) and Jørgensen et al. (2004a,c). Jørgensen et al. (2004c) found a clear anticorrelation between the abundances of $\mathrm{CO}$ and $\mathrm{N}_{2} \mathrm{H}^{+}$, which was suggested to be caused by $\mathrm{CO}$ depletion, and thereby less $\mathrm{N}_{2} \mathrm{H}^{+}$destruction, in the cold part of the envelope. It is therefore interesting to compare the $\mathrm{N}_{2} \mathrm{H}^{+}$and $\mathrm{CO}$ maps presented in this paper: as further illustrated in Fig. 10, the $\mathrm{N}_{2} \mathrm{H}^{+}$and $\mathrm{C}^{18} \mathrm{O}$ peaks are clearly anti-correlated with $\mathrm{CO}$ being present towards the central protostar. This supports the claim that the $\mathrm{C}^{18} \mathrm{O}$ maps peak toward the regions where $\mathrm{CO}$ has evaporated from the grain mantles whereas $\mathrm{N}_{2} \mathrm{H}^{+}$peaks away from the central continuum position where $\mathrm{CO}$ is frozen-out.

As discussed in Sect. 4.1, a significant fraction of the line emission is resolved out. Since the structure of the $\mathrm{C}^{18} \mathrm{O}$ emission appears relatively simple, we can calculate the emission from the envelope adopting the physical structure and CO abundances from Jørgensen et al. (2002) as shown in Fig. 11. This model is compared to "jump" models where the $\mathrm{CO}$ abundance increases at radii where the temperature is above a given threshold mimicking evaporation of CO. As can be seen from Fig. 11, the constant abundance model provides a better fit to the interferometer data: the "jump" models either overproduce the observed emission at intermediate baselines (models with $T_{\mathrm{ev}}=20 \mathrm{~K}$ and $30 \mathrm{~K}$ ) or do not produce a large increase at the shortest baselines (model with $T_{\mathrm{ev}}=40 \mathrm{~K}$ ). As discussed in Jørgensen et al. (2002, 2004c), however, although the constant $\mathrm{CO}$ abundance and jump models can successfully explain the intensities of the higher $J=2-1$ and
$J=3-2$ CO lines, they generally under-produce the low $J=1-$ 0 emission. It is therefore not unexpected that such models have problems explaining the radial variation of the observed $\mathrm{CO}$ emission.

As an alternative, Jørgensen et al. (2004b,c) suggest a "drop" model where $\mathrm{CO}$ is frozen out only in regions where the temperature is lower than the evaporation temperature $T_{\mathrm{ev}}$, while at the same time the density is higher than a given density, $n_{\text {de }}$, so that the depletion timescale is shorter than the age of the core. This is similar to the case seen for pre-stellar cores (e.g., Caselli et al. 1999; Tafalla et al. 2002; Lee et al. 2003) where $\mathrm{CO}$ is frozen out towards the core center where the density high, and the corresponding timescale for depletion thereby shorter than the lifetime of the core. Such "drop" models can explain both high and low $J$ CO lines, and the difference in abundances between more and less massive envelopes seen in Jørgensen et al. (2002), and they work very well in describing the radial variations of molecules such as $\mathrm{H}_{2} \mathrm{CO}$ (Schöier et al. 2004).

Models for the single-dish CO emission were calculated for L483 and compared to the line intensities reported in Jørgensen et al. (2002). A drop model with $n_{\mathrm{de}}=1.5 \times 10^{5} \mathrm{~cm}^{-3}$ and $T_{\text {ev }}=40 \mathrm{~K}$, an undepleted CO abundance of $2.7 \times 10^{-4}$ and depletion in the drop region by a factor 50 was found to provide good fits to the observed single-dish emission as indicated by the reduced $\chi^{2}$ for the fit as given in Table 4 . This model has been compared to the interferometer data in Fig. 11 and is found to give a good fit to the observed $\mathrm{C}^{18} \mathrm{O} 1-0$ emission at practically all baselines. Freeze-out up to a temperature of $40 \mathrm{~K}$ is needed in order not to produce too much emission at the 

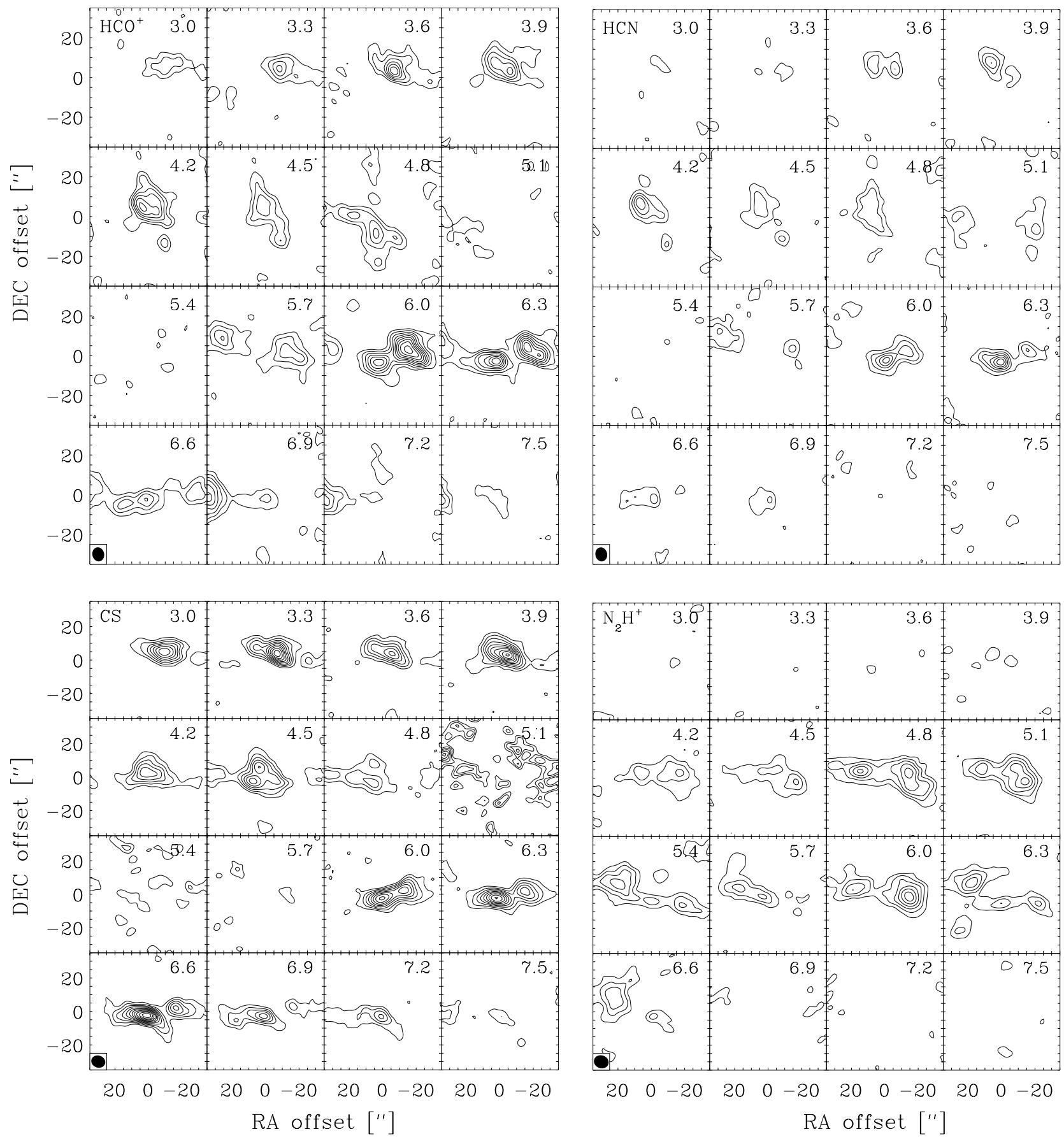

Fig. 7. Channel maps for the $\mathrm{HCO}^{+}, \mathrm{HCN}, \mathrm{CS}$ and $\mathrm{N}_{2} \mathrm{H}^{+}$emission. Contours are given in steps of $3 \sigma$.

intermediate baselines and the inclusion of an outer low density region, where the $\mathrm{CO}$ abundance increases, nicely reproduces the observed trend at the shortest baselines.

\subsubsection{Chemical implications}

The CO abundance structure has important chemical implications. As described above, distinct zones with and without depletion of $\mathrm{CO}$ also account for the characteristic shape of the $\mathrm{N}_{2} \mathrm{H}^{+}$emission. In their $\mathrm{NH}_{3}$ maps Fuller \& Wootten (2000) found a very similar structure with a "valley" devoid of emission in the north-south direction through the position of the protostar but otherwise following the large scale dust continuum emission from the SCUBA maps. Caselli et al. (2002) likewise suggested a close correlation between emission seen in $\mathrm{N}_{2} \mathrm{H}^{+}$and $\mathrm{NH}_{3}$ based on a large single-dish survey of $\mathrm{N}_{2} \mathrm{H}^{+}$ emission toward dense cores. The precursor for both $\mathrm{N}_{2} \mathrm{H}^{+}$and $\mathrm{NH}_{3}$ is $\mathrm{N}_{2}$ which due to its low binding energy does not deplete unless at very high ages and densities (e.g. Bergin \& Langer 1997; Bergin et al. 2002). $\mathrm{NH}_{3}$ maintains a balance with the overall chemical network of $\mathrm{N}$-bearing species, including $\mathrm{N}_{2} \mathrm{H}^{+}$, predominantly through reactions with $\mathrm{NH}_{4}^{+}$, and at low 
Table 4. $\chi^{2}$ for fit of models to single-dish and interferometer data.

\begin{tabular}{llll}
\hline \hline Model & & $\chi_{\text {red, }}^{2}{ }^{a}$ & $\chi_{\text {red,i }}^{2}{ }^{b}$ \\
\hline $1:$ & Jump $T_{\text {ev }}=20 \mathrm{~K}$ & 221 & 478 \\
$2:$ & Jump $T_{\mathrm{ev}}=30 \mathrm{~K}$ & 18.9 & 8.0 \\
$3:$ & Jump $T_{\mathrm{ev}}=40 \mathrm{~K}$ & 10.8 & 4.7 \\
$4:$ & Drop $T_{\mathrm{ev}}=40 \mathrm{~K} ; n_{\mathrm{de}}=1.5 \times 10^{5} \mathrm{~cm}^{-3}$ & 1.5 & 1.3 \\
$5:$ & Constant & 8.0 & 3.1 \\
\hline
\end{tabular}

${ }^{a}$ Reduced $\chi^{2}$ for fits to single-dish line intensities. ${ }^{b}$ Reduced $\chi^{2}$ for fits to interferometer data as shown in Fig. 11.

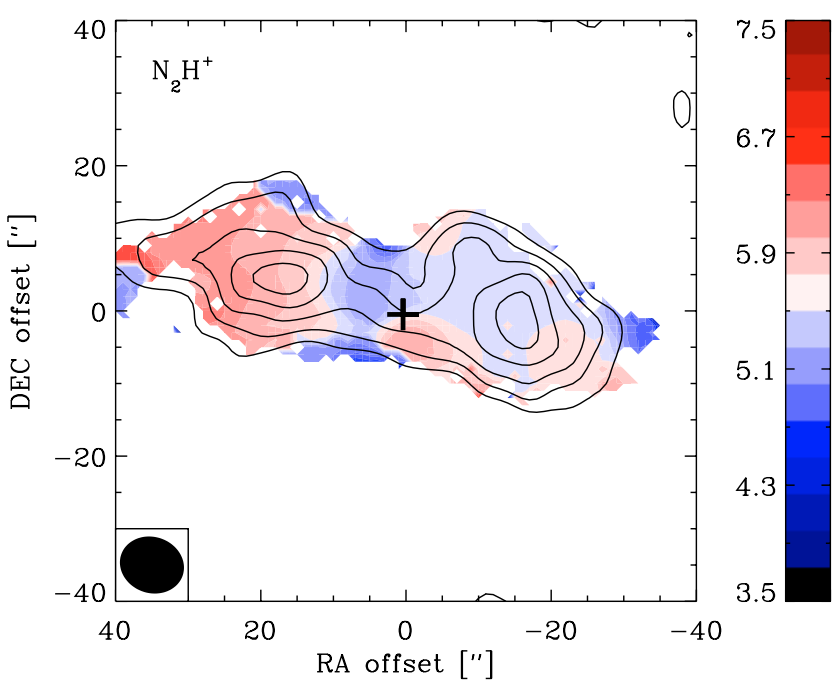

Fig. 8. The velocity field from the $\mathrm{N}_{2} \mathrm{H}^{+}$maps as colored contours compared to the integrated emission (black line contours). The velocity has been derived by fitting the 7 hyperfine components of the $\mathrm{N}_{2} \mathrm{H}^{+}$ $1-0$ line over the entire map.

(depleted) $\mathrm{CO}$ abundances these species establish a closed network. At "standard" CO abundances, reactions between $\mathrm{C}^{+}$and $\mathrm{NH}_{3}$, however, drive the nitrogen into $\mathrm{H}_{2} \mathrm{NC}^{+}$and from there to $\mathrm{CN}, \mathrm{HNC}$ and $\mathrm{HCN}$, whereas the $\mathrm{N}_{2} \mathrm{H}^{+}$is destroyed through reactions with $\mathrm{CO}$.

These trends are illustrated with a chemical model for the L483 envelope using the chemical network and approach described in Doty et al. $(2002,2004)$ and adopting the physical structure from Jørgensen et al. (2002) with CO depleted in the zone constrained by the single-dish and interferometer observations above. As shown in Fig. 12 this gives exactly an increase in $\mathrm{N}_{2} \mathrm{H}^{+}$and $\mathrm{NH}_{3}$ over the region where the $\mathrm{CO}$ is depleted. Note that although the $\mathrm{CO}$ abundance goes up again in the outermost part of the envelope and the $\mathrm{NH}_{3}$ and $\mathrm{N}_{2} \mathrm{H}^{+}$abundances consequently drop, this occurs at distances of $\approx 8000$ AU corresponding to $40^{\prime \prime}$ from the compact source in Fig. 4, which is close to the boundary of the $\mathrm{N}_{2} \mathrm{H}^{+}$emission. This is, however, probably not the main reason for the outer edge to the $\mathrm{N}_{2} \mathrm{H}^{+}$emission: it is more likely caused by the critical density of the $\mathrm{N}_{2} \mathrm{H}^{+} 1-0$ transition which is $\approx 10^{5} \mathrm{~cm}^{-3}$, a density which is also reached at this distance from the central source. The $\mathrm{N}_{2} \mathrm{H}^{+}$emission is therefore not sensitive to the outer region with low density material where $\mathrm{CO}$ is undepleted.
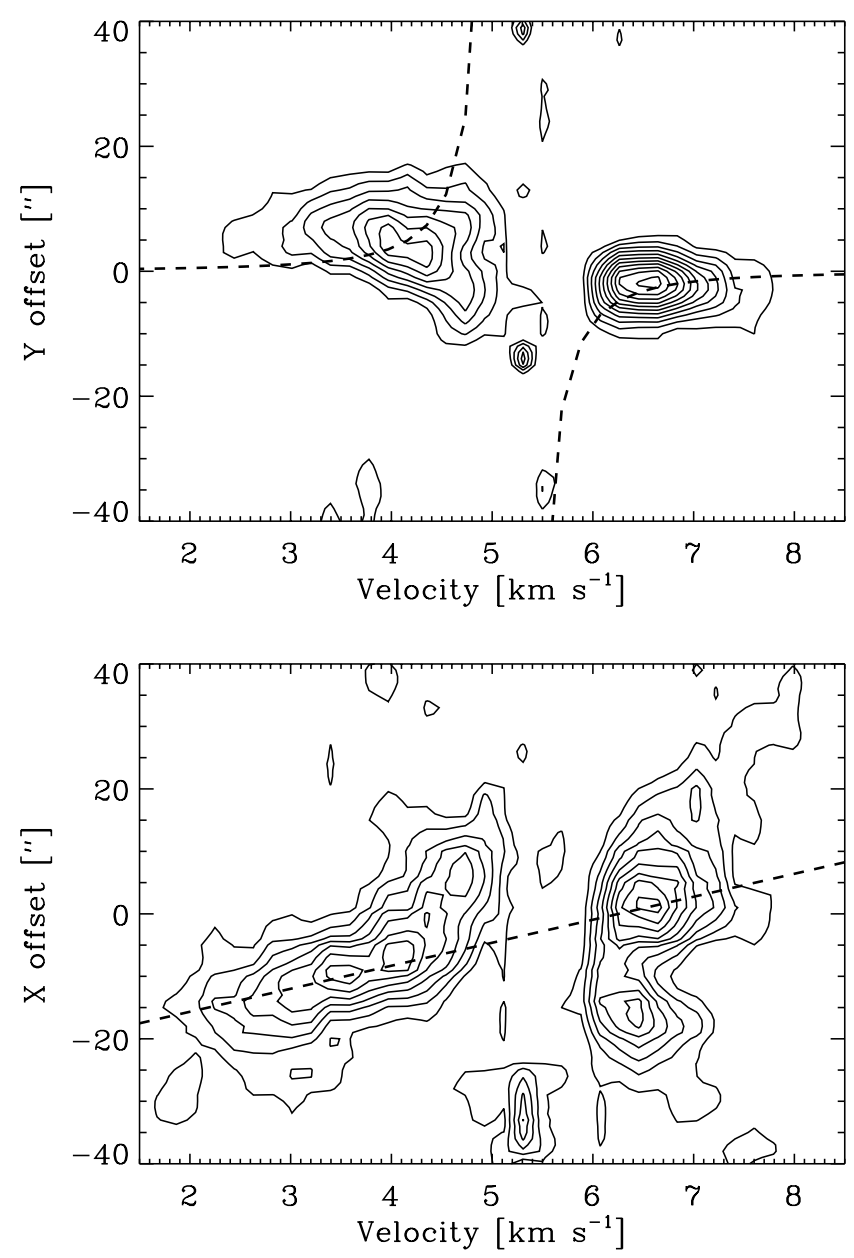

Fig. 9. Position-velocity diagram for the CS emission rotated $12^{\circ}$, so the $Y$-axis is in the direction of the velocity gradient in the dense material close to the central protostar (see also Fig. 10). The dashed lines indicate Keplerian rotation around a $1.2 M_{\odot}$ central object in the upper panel and linear expansion of $0.27 \mathrm{~km} \mathrm{~s}^{-1} \operatorname{arcsec}^{-1}$ in the lower panel.

Of course a detailed radiative transfer model for the $\mathrm{N}_{2} \mathrm{H}^{+}$ and $\mathrm{NH}_{3}$ emission could address some of these issues in more detail, such as it was done for the $\mathrm{CO}$ emission. The problem is, however, that the spherical model clearly breaks down for the scales probed by the $\mathrm{N}_{2} \mathrm{H}^{+}$emission. In fact the asymmetry may well be the reason that $\mathrm{N}_{2} \mathrm{H}^{+}$is seen in so clearly distinct zones away from the central protostar in the $\mathrm{L} 483$ case, since $\mathrm{N}_{2} \mathrm{H}^{+}$becomes marginally optically thick toward the source center in an entirely spherical core. Therefore $\mathrm{N}_{2} \mathrm{H}^{+}$will not 

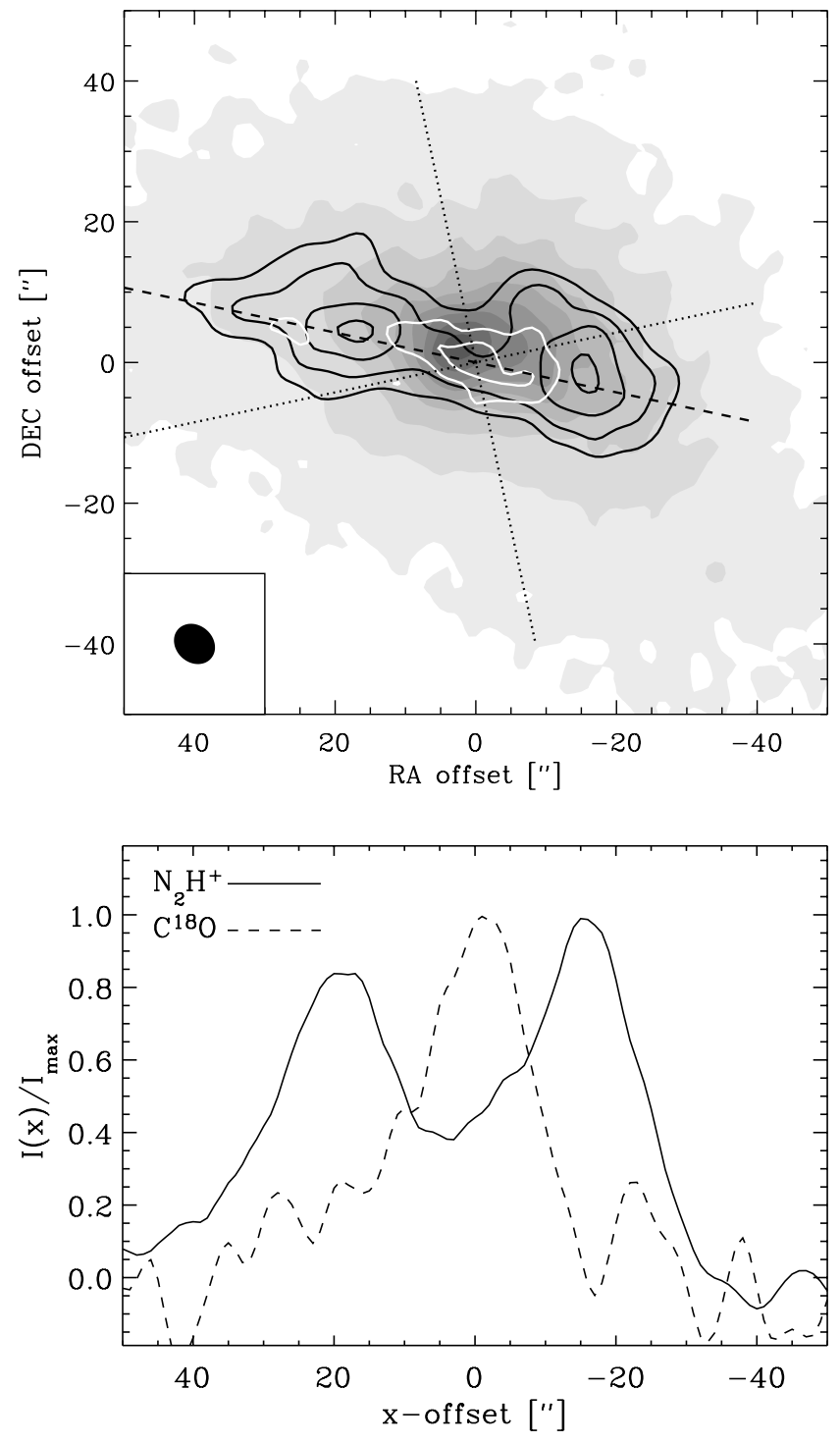

Fig. 10. Comparison between $\mathrm{N}_{2} \mathrm{H}^{+}$vs. $\mathrm{C}^{18} \mathrm{O}$ emission. Upper panel: The integrated $\mathrm{N}_{2} \mathrm{H}^{+}$and $\mathrm{C}^{18} \mathrm{O}$ emission (black and white contours) plotted over SCUBA $450 \mu \mathrm{m}$ image (grey-scale). The flattened direction of the core (i.e., $12^{\circ}$ from the east-west axis) has been indicated by the dashed line. The coordinate system with the rotation velocity gradient (Fig. 9) has been indicated by the dotted lines. Lower panel: the $\mathrm{N}_{2} \mathrm{H}^{+}$and $\mathrm{C}^{18} \mathrm{O}$ emission in a strip in the flattened direction of the core (dashed line in upper panel). Note the clear anti-correlation between the peaks in $\mathrm{C}^{18} \mathrm{O}$ and $\mathrm{N}_{2} \mathrm{H}^{+}$.

be sensitive to the innermost region where the $\mathrm{CO}$ comes off the dust grains in the symmetric case.

\subsection{UV irradiation of outflow cavity walls}

$\mathrm{HCO}^{+}$is found to be most prominent towards the outflow as is typically seen at large and small scales (e.g., Hogerheijde et al. 1998), whereas the $\mathrm{N}_{2} \mathrm{H}^{+}$emission probes the cold part of the quiescent cloud. As illustrated in Fig. 13, the $\mathrm{CN}$ emission appears in a boundary region between $\mathrm{HCO}^{+}$and $\mathrm{N}_{2} \mathrm{H}^{+}$, i.e., between the outflowing and quiescent cloud material. A possible explanation for this "borderline property" of the $\mathrm{CN}$ emission

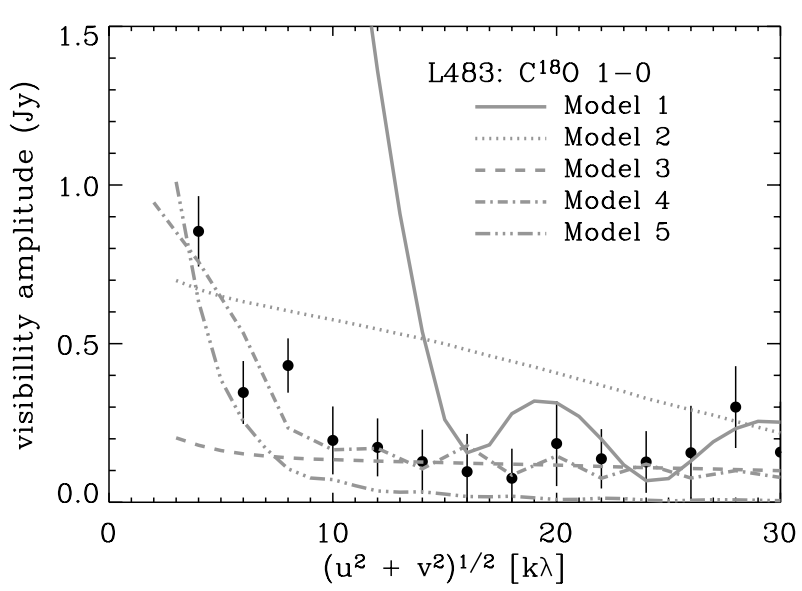

Fig. 11. Comparisons between the observed visibilities (solid circles) and model predictions (grey lines) for the $\mathrm{C}^{18} \mathrm{O}$ emission averaged over the observed line ( 4.0 to $6.5 \mathrm{~km} \mathrm{~s}^{-1}$ ) plotted vs. projected baseline length. Model 1-3 are jump models with $T_{\mathrm{ev}}$ of 20,30 and $40 \mathrm{~K}$, respectively. Model 4 is a drop model with $T_{\mathrm{ev}}=40 \mathrm{~K}$ and $n_{\mathrm{de}}=1.5 \times 10^{5} \mathrm{~cm}^{-3}$. Model 5 is a constant abundance model. Note that only model 4 is consistent with all single-dish line intensities presented in Jørgensen et al. (2002) as shown in Table 4.

could be that $\mathrm{CN}$ is enhanced in the walls of an outflow cavity probed by the $\mathrm{HCO}^{+}$emission.

Jørgensen et al. (2004c) found a clear trend between the $\mathrm{CN}$ abundances and $\mathrm{CS} / \mathrm{SO}$ abundance ratio and suggested that this might reflect the strength of ultraviolet radiation in the protostellar envelope. L483 is the source in the sample of Jørgensen et al. (2004c) with the highest ratio of $\mathrm{CN}$ abundances in the inner region relative to the outer region of the envelope (i.e., $[\mathrm{CN}]_{32} /[\mathrm{CN}]_{10}$, where $[\mathrm{CN}]_{32}$ and $[\mathrm{CN}]_{10}$ are the $\mathrm{CN}$ abundances inferred from the high excitation 3-2 lines at $340.24 \mathrm{GHz}$ and low excitation 1-0 lines at $113.49 \mathrm{GHz}$, respectively). For most sources in the sample this ratio is less than 1 indicating that the $\mathrm{CN}$ abundances are enhanced predominantly in the low density material in the outermost region of the envelope. For L483, the ratio is 2.5 indicating a higher $\mathrm{CN}$ abundance in the denser material. If the $\mathrm{CN}$ abundances are probing the strength of the UV field, this argues in favor of an internal source of the UV radiation in the case of L483.

Such correlations between the $\mathrm{CN}$ abundances and UV field have been suggested to be present in gaseous disks around more evolved class I/II objects (Dutrey et al. 1997; Qi 2001; Bergin et al. 2003; van Zadelhoff et al. 2003; Thi et al. 2004) which are known to be strong emitters of UV radiation resulting from the ongoing accretion. In the deeply embedded stages, however, the UV radiation cannot escape far out into the envelope due to the large extinctions. This will prevent direct detection of any UV flux from the central star-disk system, but this emission may still be dominant at small scales close to the central protostar. It is therefore of high interest to search for specific chemical probes that may be used to address the impact of UV in the inner envelope and thereby potentially also the ongoing accretion.

The UV radiation field may furthermore be of large importance in cavities cleared by outflows. Spaans et al. (1995), for example, suggested that the presence of high $J=6-5 \mathrm{CO}$ lines 


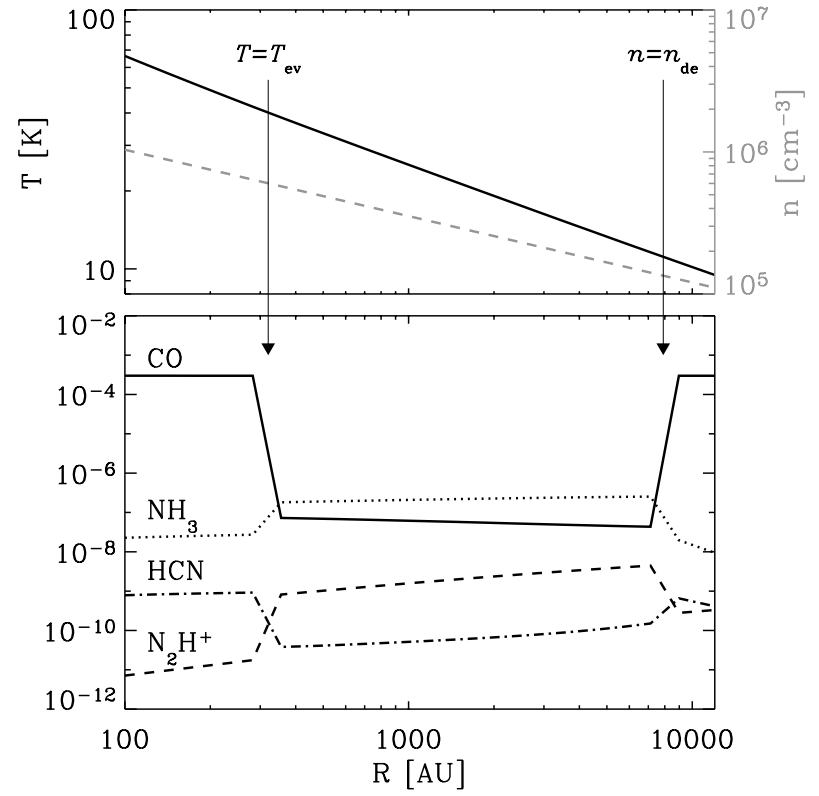

Fig. 12. Molecular abundances as function of radius for L483, adopting the chemical model appropriate for IRAS $16293-2422$ at $10^{4}$ years (Doty et al. 2004). Upper panel: the density and temperature profile for L483 shown with dashed and solid lines, respectively. Lower panel: the abundances of $\mathrm{CO}, \mathrm{NH}_{3}, \mathrm{~N}_{2} \mathrm{H}^{+}$and $\mathrm{HCN}$ from the model calculations. The $\mathrm{CO}$ abundance (solid line) structure is assumed to follow a "drop" abundance structure with an evaporation temperature, $T_{\mathrm{ev}}$, of $40 \mathrm{~K}$ and a depletion density, $n_{\mathrm{de}}$, of $1.5 \times 10^{5} \mathrm{~cm}^{-3}$ as indicated by the two vertical arrows. The $\mathrm{N}_{2} \mathrm{H}^{+}$(dashed line) and $\mathrm{NH}_{3}$ (dotted line) abundances are found to increase by one to two orders of magnitude over the region where $\mathrm{CO}$ is depleted, whereas $\mathrm{HCN}$ (dashed-dotted line) is seen to follow the $\mathrm{CO}$ abundance, dropping by a factor 5-10 over the same region.

toward embedded protostars is related to heating of material in the outflow cavity walls by the UV radiation escaping from the central protostar. The near-infrared nebulosity toward L483 (Fig. 5 and Fuller et al. 1995) suggests that such a cavity is present. Alternatively the enhanced UV radiation could be a result of the shocks associated with the L483 outflow which are directly probed by the $\mathrm{H}_{2}$ emission (Hodapp 1994; Buckle et al. 1999). Molinari et al. (2001) suggested that recombination in the post-shocked gas could illuminate outflow cavities behind Herbig-Haro objects producing a PDR resulting in diffuse $\mathrm{C}^{+}$emission seen by ISO (Molinari et al. 2001; Molinari $\&$ Noriega-Crespo 2002).

To get a handle on this possibility a simple estimate of the extent of the $\mathrm{CN}$ emitting region can be made: the $\mathrm{CN}$ emission is unresolved in its transverse direction, meaning that the extent of the emission must be $\lesssim 5^{\prime \prime}$. In the model for the L483 envelope from Jørgensen et al. (2002) the density at $20^{\prime \prime}$ from the protostar is $n\left(\mathrm{H}_{2}\right)=3 \times 10^{5} \mathrm{~cm}^{-3}$. Introducing the extent of the CN emission as $l \lesssim 5^{\prime \prime}$, an upper limit to the column density of the $\mathrm{CN}$ emitting region can be estimated:

$N_{\mathrm{H}_{2}} \lesssim 4 \times 10^{21} \mathrm{~cm}^{-2}\left(\frac{n\left(\mathrm{H}_{2}\right)}{3 \times 10^{5} \mathrm{~cm}^{-3}}\right)\left(\frac{l}{5^{\prime \prime}}\right)$

or a visual extinction, $A_{\mathrm{V}} \lesssim 4$. Models of photon dominated regions (e.g., Jansen et al. 1995; Sternberg \& Dalgarno 1995)
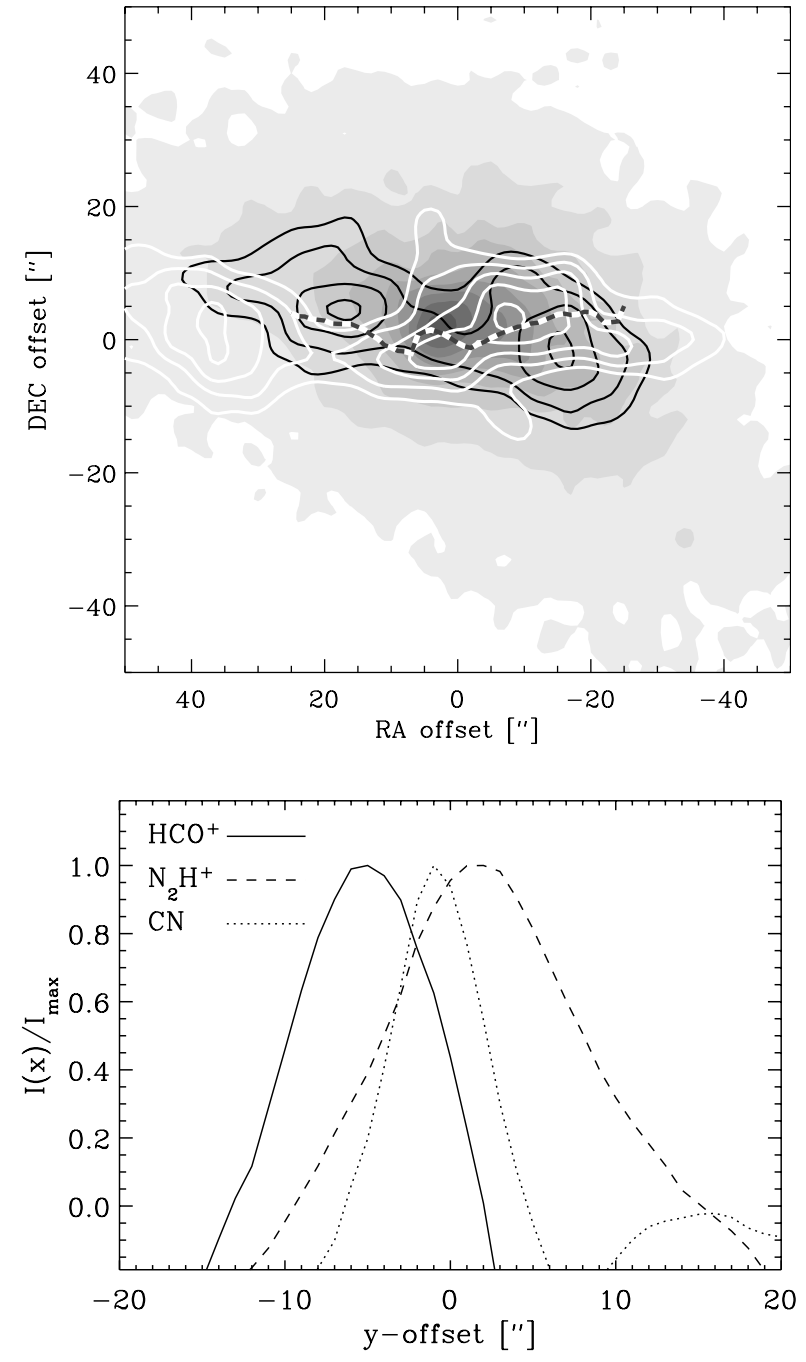

Fig. 13. Comparison between $\mathrm{HCO}^{+}, \mathrm{N}_{2} \mathrm{H}^{+}$and $\mathrm{CN}$ emission. Upper panel: SCUBA $450 \mu \mathrm{m}$ image with $\mathrm{HCO}^{+}$emission and $\mathrm{N}_{2} \mathrm{H}^{+}$emission as white and black solid line contours, respectively, and the vertical centroid of the $\mathrm{CN}$ emission overplotted (black/white dashed line). Lower panel: emission of the three species in the vertical direction averaged over the eastern outflow lobe (offsets between 10 and $25^{\prime \prime}$ in the coordinate system aligned with the flattened direction of the core (dashed lines in Fig. 10).

indicate that this is the extinction range over which $\mathrm{CN}$ would be enhanced predominantly due to photodissociation of HCN. Alternative explanations such as excitation effects appear less likely, since $\mathrm{CN}$ is the only molecule showing this peculiar morphology.

If $\mathrm{CN}$ in fact is tracing the interface region between the outflow and the quiescent core probed by $\mathrm{HCO}^{+}$and $\mathrm{N}_{2} \mathrm{H}^{+}$, respectively, this should also be reflected in the observed velocity field of the three species. As discussed above the velocity field probed by $\mathrm{N}_{2} \mathrm{H}^{+}$shows a red-blue spatial asymmetry aligned with that seen in $\mathrm{HCO}^{+}$, suggesting that the "quiescent" material probed by $\mathrm{N}_{2} \mathrm{H}^{+}$is being accelerated by the outflow. Figure 14 compares the velocity field in the eastern lobe of the three species along the flattened direction of the core (dashed line in Fig. 10). The $\mathrm{HCO}^{+}$does indeed show the largest velocity gradient across the region with less steep increases in $\mathrm{CN}$ 


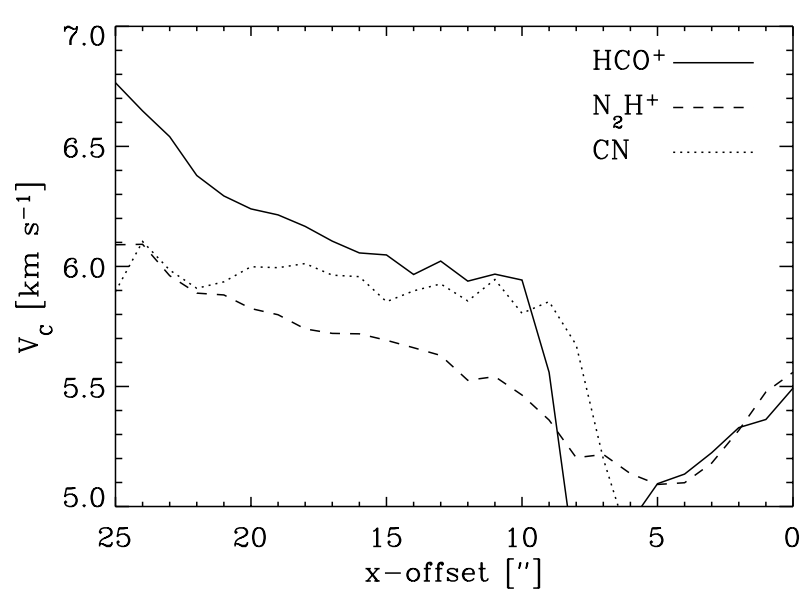

Fig. 14. Velocity field in $\mathrm{HCO}^{+}, \mathrm{N}_{2} \mathrm{H}^{+}$and $\mathrm{CN}$ emission: average centroid velocity of the three species in strips perpendicular to the outflow axis across the eastern part of the core.

and $\mathrm{N}_{2} \mathrm{H}^{+}$toward the lower densities in the core. Again $\mathrm{CN}$ is seen to be intermediate between the outflowing and quiescent material in support of the suggested scenario.

\section{Conclusions}

We have presented an analysis of the physical and chemical properties of the class 0 protostar L483-mm on 500-1000 AU scales. This paper demonstrates how the 1D envelope model derived from single-dish continuum and line data can be applied to the interpretation of the millimeter interferometer observations. The main conclusions are as follows:

1. The continuum emission at $3 \mathrm{~mm}$ is well-fit by the envelope model from Jørgensen et al. (2002). In contrast to the case for other recently studied class 0 objects (e.g., NGC 1333IRAS 2A, L1448-C and IRAS 16293-2422; Jørgensen et al. 2004a; Schöier et al. 2004), an additional point source (e.g., a disk) is not needed to fit the observed continuum emission. Assuming an optically thin $30 \mathrm{~K}$ disk, this puts an upper limit to the disk mass of $0.04 M_{\odot}$.

2. The $\mathrm{C}^{18} \mathrm{O}$ emission is found to be well-described in a "drop abundance" model (Jørgensen et al. 2004b,c) where the abundance is high in the outermost regions (densities lower than $1.5 \times 10^{5} \mathrm{~cm}^{-3}$ ) and innermost (temperatures higher than $40 \mathrm{~K}$ ) parts of the envelope but frozen out in between.

3. $\mathrm{N}_{2} \mathrm{H}^{+}$and $\mathrm{C}^{18} \mathrm{O}$ are found to be clearly anti-correlated, with $\mathrm{C}^{18} \mathrm{O}$ centered on the central continuum source whereas $\mathrm{N}_{2} \mathrm{H}^{+}$has two distinct peaks away from the central star. This is interpreted as the combined effects of $\mathrm{CO}$ freeze-out at low temperatures and destruction of $\mathrm{N}_{2} \mathrm{H}^{+}$by reactions with $\mathrm{CO}$. The $\mathrm{N}_{2} \mathrm{H}^{+}$emission resembles that of $\mathrm{NH}_{3}$, previously reported by Fuller \& Wootten (2000), which can be understood if $\mathrm{NH}_{3}$ is destroyed through reactions with $\mathrm{C}^{+}$ when the $\mathrm{CO}$ abundance is high, and as clearly illustrated by a chemical model for the L483 envelope.

4. $\mathrm{HCN}$ and $\mathrm{CS}$ emission probe the dense material close to the central protostar. A velocity gradient perpendicular to the outflow propagation direction, also recognized in previous
$\mathrm{C}_{3} \mathrm{H}_{2}$ observations by Park et al. (2000), is interpreted as rotation around $1 M_{\odot}$ central protostar.

5. $\mathrm{CN}$ is found to trace a boundary between the quiescent material probed by $\mathrm{N}_{2} \mathrm{H}^{+}$and the larger scale outflow seen in $\mathrm{HCO}^{+}$. A possible explanation is that $\mathrm{CN}$ probes material in the outflow cavity walls (seen as an infrared nebula in 2MASS $K_{\mathrm{s}}$ images) where its abundance is enhanced as a result of UV irradiation. This is further supported by the observed velocity field: the gradient introduced by the outflow is seen in both $\mathrm{HCO}^{+}, \mathrm{CN}$ and $\mathrm{N}_{2} \mathrm{H}^{+}$, with $\mathrm{HCO}^{+}$ showing the largest velocities relative to the systemic velocity followed by $\mathrm{CN}$ (in the cavity walls) and $\mathrm{N}_{2} \mathrm{H}^{+}$(in the core material). This also suggests that a clear interaction between the outflowing and quiescent material around L483 is taking place. Possibly the outflow is in the process of dispersing the protostellar core, causing its characteristic asymmetric shape.

This paper illustrates the potential of high-resolution millimeter interferometer observations for addressing the spatial variations in the chemistry around protostellar objects. In particular, future high resolution observations from the SMA, CARMA and eventually ALMA will confirm or reject the drop abundance model through imaging of the radial structure of the emission from high excitation lines of, e.g., $\mathrm{C}^{18} \mathrm{O}$, probing more uniquely the higher temperatures and densities in the envelope. Also high resolution observations of high excitation lines of $\mathrm{CN}$ will make it possible to further address the importance of UV radiation in the inner envelope close to the central protostar and confirm the relation between $\mathrm{CN}$ abundances and strength of the UV field. In this context direct imaging of lines of, e.g., atomic carbon in the high frequency windows will also be important to understand the specific chemical effects. Such observations will become feasible with the SMA and ALMA. An additional test would be deep mid-infrared Spitzer observations: for example, deep imaging of the thermal emission in the outflow cavities and searches for established probes of the UV field such as the presence and properties of specific emission lines and PAH features, provide complementary information which could be used to establish a more detailed 2D model of complex protostellar sources such as L483.

Acknowledgements. Ewine van Dishoeck, Michiel Hogerheijde, Fredrik Schöier and Geoff Blake are thanked for fruitful discussions and comments on the manuscript. Michiel Hogerheijde and Floris van der Tak are further acknowledged for making their radiative transfer code publically available. Steve Doty is thanked for the use of his chemical code. This research was funded by a NOVA network 2 Ph.D. stipend.

\section{References}

Alves, J. F., Lada, C. J., \& Lada, E. A. 2001, Nature, 409, 159

Bachiller, R., \& Pérez Gutiérrez, M. 1997, ApJ, 487, L93

Beltrán, M. T., Estalella, R., Anglada, G., Rodríguez, L. F., \& Torrelles, J. M. 2001, AJ, 121, 1556

Bergin, E., Calvet, N., D’Alessio, P., \& Herczeg, G. J. 2003, ApJ, 591, L159

Bergin, E. A., Alves, J., Huard, T., \& Lada, C. J. 2002, ApJ, 570, L101 
Bergin, E. A., Ciardi, D. R., Lada, C. J., Alves, J., \& Lada, E. A. 2001, ApJ, 557, 209

Bergin, E. A. \& Langer, W. D. 1997, ApJ, 486, 316

Buckle, J. V., Hatchell, J., \& Fuller, G. A. 1999, A\&A, 348, 584

Caselli, P., Benson, P. J., Myers, P. C., \& Tafalla, M. 2002, ApJ, 572, 238

Caselli, P., Walmsley, C. M., Tafalla, M., Dore, L., \& Myers, P. C. 1999, ApJ, 523, L165

Doty, S. D., Schöier, F. L., \& van Dishoeck, E. F. 2004, A\&A, 418, 1021

Doty, S. D., van Dishoeck, E. F., van der Tak, F. F. S., \& Boonman, A. M. S. 2002, A\&A, 389, 446

Dutrey, A., Guilloteau, S., \& Guelin, M. 1997, A\&A, 317, L55

Fuller, G. A. \& Wootten, A. 2000, ApJ, 534, 854

Fuller, G. A., Lada, E. A., Masson, C. R., \& Myers, P. C. 1995, ApJ, 453,754

Harvey, D. W. A., Wilner, D. J., Lada, C. J., et al. 2001, ApJ, 563, 903

Harvey, D. W. A., Wilner, D. J., Myers, P. C., Tafalla, M., \& Mardones, D. 2003, ApJ, 583, 809

Hatchell, J., Fuller, G. A., \& Ladd, E. F. 1999, A\&A, 344, 687

Hodapp, K. 1994, ApJS, 94, 615

Hogerheijde, M. R. 2001, ApJ, 553, 618

Hogerheijde, M. R., van Dishoeck, E. F., Blake, G. A., \& van Langevelde, H. J. 1998, ApJ, 502, 315

Hogerheijde, M. R., van Dishoeck, E. F., Salverda, J. M., \& Blake, G. A. 1999, ApJ, 513, 350

Jansen, D. J., Spaans, M., Hogerheijde, M. R., \& van Dishoeck, E. F. 1995, A\&A, 303, 541

Jørgensen, J. K., Schöier, F. L., \& van Dishoeck, E. F. 2002, A\&A, 389, 908

Jørgensen, J. K., Hogerheijde, M. R., van Dishoeck, E. F., Blake, G. A., \& Schöier, F. L. 2004a, A\&A, 413, 993
Jørgensen, J. K., Schöier, F. L., \& van Dishoeck, E. F. 2004b, A\&A, submitted

Jørgensen, J. K., Schöier, F. L., \& van Dishoeck, E. F. 2004c, A\&A, 416, 603

Lee, J.-E., Evans, N. J., Shirley, Y. L., \& Tatematsu, K. 2003, ApJ, 583,789

Looney, L. W., Mundy, L. G., \& Welch, W. J. 2000, ApJ, 529, 477

Maret, S., Ceccarelli, C., Caux, E., et al. 2004, A\&A, 416, 577

Molinari, S., \& Noriega-Crespo, A. 2002, AJ, 123, 2010

Molinari, S., Noriega-Crespo, A., \& Spinoglio, L. 2001, ApJ, 547, 292

Park, Y., Panis, J., Ohashi, N., Choi, M., \& Minh, Y. C. 2000, ApJ, 542, 344

Qi, C. 2001, Ph.D. Thesis

Schöier, F. L., Jørgensen, J. K., van Dishoeck, E. F., \& Blake, G. A. 2002, A\&A, 390, 1001

Schöier, F. L., Jørgensen, J. K., van Dishoeck, E. F., \& Blake, G. A. 2004, A\&A, 418, 185

Scoville, N. Z., Carlstrom, J. E., Chandler, C. J., et al. 1993, PASP, 105,1482

Shirley, Y. L., Evans, N. J., \& Rawlings, J. M. C. 2002, ApJ, 575, 337

Spaans, M., Hogerheijde, M. R., Mundy, L. G., \& van Dishoeck, E. F. 1995, ApJ, 455, L167

Sternberg, A., \& Dalgarno, A. 1995, ApJS, 99, 565

Tafalla, M., \& Myers, P. C. 1997, ApJ, 491, 653

Tafalla, M., Myers, P. C., Caselli, P., Walmsley, C. M., \& Comito, C. 2002, ApJ, 569, 815

Tafalla, M., Myers, P. C., Mardones, D., \& Bachiller, R. 2000, A\&A, 359,967

Thi, W.-F., van Zadelhoff, G.-J., \& van Dishoeck, E. F. 2004, A\&A, submitted

van Zadelhoff, G.-J., Aikawa, Y., Hogerheijde, M. R., \& van Dishoeck, E. F. 2003, A\&A, 397, 789 